ఠ

\title{
Neuraxial and peripheral nerve blocks in patients taking anticoagulant or thromboprophylactic drugs: challenges and solutions
}

This article was published in the following Dove Press journal:

Local and Regional Anesthesia

4 August 2015

Number of times this article has been viewed

Jinlei Li

Thomas Halaszynski

Department of Anesthesiology, Yale University, Yale New Haven Hospital, New Haven, CT, USA
Correspondence: Thomas Halaszynski Department of Anesthesiology,

Yale University School of Medicine,

333 Cedar Street, PO Box 20805I,

New Haven, CT 06520, USA

$\mathrm{Tel}+\mathrm{I} 2037852804$

Fax +I 2037856664

Email thomas.halaszynski@yale.edu
Abstract: Incidence of hemorrhagic complications from neuraxial blockade is unknown, but classically cited as 1 in 150,000 epidurals and 1 in 220,000 spinals. However, recent literature and epidemiologic data suggest that for certain patient populations the frequency is higher ( 1 in 3,000). Due to safety concerns of bleeding risk, guidelines and recommendations have been designed to reduce patient morbidity/mortality during regional anesthesia. Data from evidence-based reviews, clinical series and case reports, collaborative experience of experts, and pharmacology used in developing consensus statements are unable to address all patient comorbidities and are not able to guarantee specific outcomes. No laboratory model identifies patients at risk, and rarity of neuraxial hematoma defies prospective randomized study so "patientspecific" factors and "surgery-related" issues should be considered to improve patient-oriented outcomes. Details of advanced age, older females, trauma patients, spinal cord and vertebral column abnormalities, organ function compromise, presence of underlying coagulopathy, traumatic or difficult needle placement, as well as indwelling catheter(s) during anticoagulation pose risks for significant bleeding. Therefore, balancing between thromboembolism, bleeding risk, and introduction of more potent antithrombotic medications in combination with regional anesthesia has resulted in a need for more than "consensus statements" to safely manage regional interventions during anticoagulant/thromboprophylactic therapy.

Keywords: antithrombotics, novel oral anticoagulant, regional, neurologic dysfunction, hematoma, peripheral nerve blockade

\section{Introduction}

Searching for an ideal anticoagulant and thromboprophylactic medication is transitioning toward agents with improved efficacy, better patient safety profile(s), reduced bleeding potential, and cost lowering benefits. ${ }^{1,2}$ This search presents challenges for clinicians involved in neuraxial, superficial, and deep peripheral nerve/nerve plexus blockade, collectively identified as regional anesthesia (RA). Newly added coagulationaltering therapies creates additional confusion to understanding commonly used medications affecting coagulation in conjunction with RA. However, there is also promising new evidence that novel oral anticoagulants (NOACs) acting as inhibitors of thrombin/factor IIa or factor Xa may be more effective in thromboprophylaxis and preventing deep vein thrombosis (DVT). In addition, NOACs with fixed-dose administration, reduced need for monitoring, fewer requirements of dose adjustment, and more favorable pharmacokinetics and pharmacodynamics are likely to streamline perioperative management, simplify transitioning of agents, diversify "bridging therapy" options, and reduce therapy costs. ${ }^{1,3}$ 
Synopsis of opinions and evidence-based recommendations in this article are based upon recommendations/ guidelines from several respected agencies including American Society of Regional Anesthesia (ASRA), American College of Chest Physicians (ACCP), and European Society of Regional Anesthesia (ESRA) among others. Investigations of large-scale randomized controlled trials studying RA in conjunction with coagulation-altering medications are not feasible due to: 1) medical-legal considerations and 2) since nerve tissue compromise from hematoma development is rare, very large sample sizes are required. Therefore, attempts at striking a balance between catastrophic thromboembolic events and hemorrhagic complications will remain a strategy for clinicians practicing RA in the perioperative environment.

Guidelines for practicing RA in conjunction with patients taking anticoagulants/thromboprophylactics are based on best available information and evidence-based recommendations with goals to standardize hospital-based medical practice, optimize patient outcomes, and promote quality patient care. However, no specific clinical outcome can be guaranteed from the suggested guidelines. In addition, variation from evidencebased recommendations based on best practices should not be deemed deviation from "standard of care". For example, ASRA and ESRA experiences can be markedly different under certain clinical situations. ${ }^{4-6}$ Therefore, understanding the complexity of this issue is essential, and raises concern on how to best follow the "consensus statements" due to clinical circumstances and changing patient comorbidities.

To manage patients on anticoagulant/thrombolytic therapy, understanding pharmacokinetic and pharmacodynamic medication interactions is necessary (especially recently introduced medications). Information to guide clinical practice such as timing of anticoagulant/thromboprophylactic administration and appropriate safety/timing of performing invasive procedures has not been satisfactorily or scientifically addressed. Necessary information to answer basic clinical parameters would be medication elimination half-life $\left(T_{1 / 2}\right)$ and time to maximum plasma concentration $\left(T_{\max }\right)$ along with serious considerations for any organ dysfunction (renal, hepatic, and cardiac systems).

Basic pharmacokinetic rules to observe include the following: 1) 8-hour interval-rule for neuraxial blocks (time between end of surgery/procedure to peak plasma level of postoperative anticoagulants); 2) not performing neuraxial/ deep-peripheral nerve blocks (PNBs) or catheter removal until at least $2-T_{1 / 2}\left(T_{1 / 2}\right.$ depending on renal and hepatic function) after last anticoagulant administration for optimal risk/ benefit ratio ( $25 \%$ pharmacodynamic efficacy or being more conservative with $5-T_{1 / 2}[3.125 \%$ anticoagulant in circulation] in high-risk patients or from new anticoagulants with limited clinical experience); 3) following catheter removal/ neuraxial and deep needle puncture, next anticoagulant administration should be based on the time required for medication to reach maximum activity, which is calculated as: hemostasis time (6-8 hours with no known coagulopathy) minus time to achieve peak plasma level (ie, drug X requires 4 hours to attain peak plasma level, then it can be administered 2-4 hours post-RA); and 4) clinical vigilance during initial hours/days following neuraxial and deep RA for evidence of compromising hematoma formation. ${ }^{7}$

Coagulation-altering medications used for prophylactic-to-therapeutic anticoagulation present a spectrum of controversy related to clinical effects, surgery, and performance of RA, including PNB, especially in the medically compromised. They range from low risk for performing neuraxial procedures during acetylsalicylic acid (aspirin) therapy to high risk for preforming such interventions with therapeutic anticoagulation. Combining two or more coagulation-altering medications can lead to adverse clotforming activity, increases the risk of hematoma development, and raises concern of neurologic compromise when RA is planned. Studies showed that combining two hemostasisaltering compounds have an additive or synergistic effect on coagulation, with increased risk of bleeding. ${ }^{8-10}$ Several classes of hemostasis-altering medications encountered in clinical practice are reviewed in Table 1.

\section{Herbal medications and antiplatelet drugs}

Many surgical patients use herbal medications with potential for complications in the perioperative period because of polypharmacy and physiological alterations. Some complications include bleeding from garlic, ginkgo, and ginseng, along with the potential interaction between ginseng and warfarin. ${ }^{11}$ It remains important that clinicians be familiar with literature on herbal medications because of new discoveries about effects of herbal medications in humans. However, herbal medications, when administered independent to other coagulation-altering therapy is not a contraindication to performing RA.

Aspirin and other nonsteroidal anti-inflammatory drugs (NSAIDs) when administered alone during the perioperative period are not considered a contraindication to RA. However, there are reports of spontaneous bleeding in patients on aspirin alone with no additional risk factors following neuraxial procedures. ${ }^{12-14}$ In patients on combination therapy with medications affecting more than one coagulation 
Table I Classes of hemostasis-altering medications

Herbal medications

- Garlic

- Ginkgo

- Ginseng

Antiplatelet medications

- Aspirin (ASA)

- Nonsteroidal anti-inflammatory drugs (NSAIDs)

- Thienopyridine derivatives (ticlopidine, clopidogrel)

- Platelet glycoprotein (GP) Ilb/lla inhibitors (GPIlb/llla receptor antagonists) Unfractionated heparin (UFH) intravenous and subcutaneous Low-molecular-weight heparin (LMWH)

Vitamin $\mathrm{K}$ antagonists: warfarin

Thrombin (factor lla) inhibitors

- Desirudin

- Lepirudin

- Bivalirudin

- Argatroban

- Dabigatran

Factor Xa inhibitors

- Fondaparinux

- Rivaroxaban

- Apixaban

- Edoxaban

- Betrixaban (in development)

- Darexaban (development discontinued)

- Otamixaban (development discontinued)

Thrombolytic and fibrinolytic medications

- Tissue plasminogen activator (tPA)

- Streptokinase

- Urokinase

- Anistreplase

mechanism, clinicians should be cautious about neuraxial and deep-PNB techniques due to increased risks of bleeding. ${ }^{15-17}$ Cyclooxygenase-2 inhibitors have shown minimal effect on platelet function, considered safe for patients receiving RA, and without additive effects in the presence of anticoagulation therapy. ${ }^{18,19}$

Antiplatelet medications including thienopyridine derivatives and platelet glycoprotein (GP) IIb/IIIa antagonists can have diverse pharmacologic effects on coagulation and platelet function. Such variable differences cause difficulty when considering RA, as there are no acceptable tests that will guide antiplatelet therapy. Therefore, preoperative assessment should search for health considerations that contribute to altered coagulation (bruising easily/excessive bleeding, female sex with increased age). Risk of hematoma formation with ticlopidine/clopidogrel and the GPIIb/IIIa antagonists in combination with RA is unknown, therefore, management is based on labeling and surgical reviews: 1) time between discontinuation of therapy and neuraxial/deep-PNB is 14 days for ticlopidine and 5-7 days for clopidogrel; 2) if performing RA is indicated before completing suggested time interval(s), then normalization of platelet function should be demonstrated; and 3) platelet GPIIb/IIIa inhibitors exert effect on platelet aggregation and time to normal platelet aggregation is 24-48 hours for abciximab and 4-8 hours for eptifibatide and tirofiban following discontinuation. ${ }^{20}$ However, deep-PNB/neuraxial techniques should be avoided until demonstrating platelet function recovery. GPIIb/IIIa antagonists are contraindicated within 4 weeks of surgery, and patients need to be monitored neurologically if such medications are administered in the postoperative period subsequent to neuraxial/deep-PNB.

\section{Intravenous and subcutaneous heparin Unfractionated heparin}

Anesthetic management of patients receiving unfractionated heparin (UFH) should start with review of medical records to determine any concurrent medication that influences clotting mechanism(s). There is no contraindication to RA with 5,000 units (U) twice daily subcutaneous (SQ) UFH (prophylaxis). Risks of bleeding are reduced by delaying heparinization until block completion, but may be increased in debilitated patients following prolonged heparin therapy. Safety of neuraxial/ deep-PNB in those receiving UFH in doses $>10,000 \mathrm{U} /$ day or more than twice-daily dosing has not been determined, and thrice-daily UFH can lead to increased risk of bleeding. ${ }^{21}$ It is suggested that risk-to-benefit ratio of thrice-daily UFH in combination with RA be individually assessed and techniques that detect evidence of neuro-deficits (enhanced neurologic monitoring and local solutions minimizing motor blockade) be incorporated.

Heparin-induced thrombocytopenia (HIT) can occur during administration, so it is recommended that patients receiving heparin $>4$ days be assessed (ie, platelet count) before deep$\mathrm{PNB}$ /neuraxial blockade or catheter removal. An investigation of 665 patients receiving SQ-UFH or low-molecular-weight heparin (LMWH) for thromboprophylaxis following hip surgery reported $2.7 \%$ incidence of HIT in those receiving UFH and $0 \%$ in patients receiving LMWH. ${ }^{22}$ This study defined HIT as a decrease in platelet count in the presence of heparindependent antiplatelet antibodies. Other systemic reviews have shown lower risk of HIT associated with LMWH compared to UFH, with a $76 \%$ relative-risk reduction for HIT. ${ }^{23,24}$

Intraoperative heparin anticoagulation during vascular surgery combined with neuraxial anesthesia is acceptable with the following: 1) avoiding neuraxial in patients with coagulopathies; 2) delaying heparinization for 1 hour 
following nontraumatic needle placement (German Society of Anesthesiology suggests 4-6 hours); 3) using concentration of local that permits neurological evaluation; 4) monitor patients postoperatively for evidence of neuro-deficits; 5) removing neuraxial catheter 2-4 hours following last heparin dose (German Society of Anesthesiology suggests 4-6 hours); and 6) assessing coagulation status, then resume heparin in 1 hour following catheter removal.

Occurrence of a bloody/difficult neuraxial blockade in vascular surgery and plan for intraoperative heparin can increase bleeding risk. However, there are no data to support "mandatory" surgery cancellation. Therefore, a risk-benefit decision should be conducted with the surgeon and 1) using low-dose anticoagulation (5,000 U) and delay its administration for 1-2 hours; 2) avoiding full intraoperative heparin for 6-12 hours; or 3) postponing surgery to the next day should be considered. Alternatively, an epidural catheter placement could be placed the evening before surgery. At therapeutic doses, UFH administration should be interrupted at least 4 hours before performing neuraxial procedures and/or removal of neuraxial catheter. In situations of full anticoagulation (ie, cardiac surgery), risk of a hematoma is unknown when combined with neuraxial techniques. Therefore, if using neuraxial anesthesia during cardiac surgery, it is suggested to monitor neurologic function and select local solutions that minimize motor blockade in order to facilitate detection of neuro-deficits.

\section{Low-molecular-weight heparin}

Properties of LMWH differ from UFH in the following ways: 1) the lack of monitoring of anticoagulant response (anti-Xa level not predictive of risk), 2) prolonged $T_{1 / 2}$ (SQ elimination $T_{1 / 2} 3-6$ hours or three to four times $T_{1 / 2}$ of UFH), 3) anti-Xa activity present 12 hours postinjection (dose independent), and 4) unpredictable response to protamine. ${ }^{25,26}$ Long-term therapy with LMWH can have accumulation of anti-Xa activity along with fibrinolysis, $T_{1 / 2}$ increases with renal compromise, and there are no studies comparing efficacy/ safety of one LMWH to another. ${ }^{25}$ There is increased risk of hematoma with concomitant use of hemostasis-altering medications and LMWH (dosage irrelevant). Presence of blood during needle/catheter placement does not necessitate canceling surgery, but LMWH therapy in this setting should be delayed 24 hours.

Altered coagulation can occur with preoperative LMWH thromboprophylaxis, and it is recommended that deep-PNB/ neuraxial placement be delayed 10-12 hours after last dose. In patients receiving preoperative therapeutic $\mathrm{LMWH}$, delay of 24 hours (minimum) is recommended to ensure adequate hemostasis at time of RA procedure. It is not recommended to perform neuraxial/deep-PNB techniques in patients receiving LMWH 2 hours preoperatively, because needle placement would occur at peak anticoagulant activity.

Management of postoperative LMWH thromboprophylaxis and neuraxial/deep-PNB techniques is based upon: 1) time-to-first postoperative dose, 2) total daily dose, and 3) dosing schedule. Neuraxial/deep-PNB can be safely performed with LMWH single-daily dosing with first dose administered 6-8 hours postoperatively after confirming adequate hemostasis and second dose no sooner than 24 hours later. Catheters may be maintained, but should be removed minimum 10-12 hours following the last dose of LMWH and subsequent dosing a minimum of 2 hours after catheter removal. Additional hemostasis-altering medications should be avoided. Twice-daily postoperative LMWH is associated with increased risk of hematoma formation, so first dose should be delayed 24 hours postoperatively along with evidence of adequate hemostasis. Catheters should be removed before twice-daily LMWH initiation and subsequent dosing delayed 2 hours postcatheter removal.

\section{Vitamin K antagonists}

Warfarin-sodium interferes with vitamin K-dependent clotting factors (II, VII, IX, X), is routinely stopped 4-5 days preoperatively ( \pm bridging therapy), and the international normalized ratio (INR) should be within reference-range prior to initiation of RA. If thromboprophylaxis is planned postoperatively and analgesia with neuraxial or deep perineural catheter(s) has been initiated, INR should be monitored on a daily basis. In addition to laboratory testing, neurologic assessment of sensory and motor function must be performed and concentration/intensity of local anesthetic blockade adjusted/lowered to facilitate neurologic evaluation. It is also recommended that neuraxial/deep perineural catheter manipulation occur when INR is $<1.5$ (derived from studies correlating hemostasis with clotting factor activity levels of $>40 \%$ ) and to continue neurologic checks 24 hours following catheter removal. ${ }^{11}$

With INR $>1.5$ but $<3$, use caution when removing indwelling catheters and review medication records for concomitant therapy that influences hemostasis without affecting INR (NSAIDs, heparin). Assessment of neurologic status after catheter removal is required and continued until INR has stabilized (levels $<1.5$ ). In patients with an INR $>3$, warfarin should be held/reduced with concurrent indwelling neuraxial and deep perineural catheters. There are no recommendations regarding management of catheters in patients 
receiving therapeutic levels of warfarin in conjunction with neuraxial/deep-PNB catheters.

Warfarin administration and correlation between vitamin K-dependent factor levels and INR critical to RA management are that 1) INR can drop or approach normal levels 1-3 days following warfarin discontinuation (reflecting increase of factor VII activity); however, 2) inadequate hemostasis remains possible despite decreased INR (factors-II with longer $T_{1 / 2}$ still $<40 \%$ activity). ${ }^{27}$ Therefore, adequate levels of II, VII, IX, and X ( $\geq 40 \%$ activity) should be present reflecting adequate INR within reference limits.

\section{New anticoagulants}

Administration of medications targeting the hemostatic system in combination with RA must consider risk to benefit, and until investigational series become available, management recommendations should be made according to dosing plan/timing, agent $T_{1 / 2}$, exaggerated response in patients with comorbidities, and risks from concomitant medications affecting coagulation. Despite potential for more efficacious clinical effects with these newer agents, incorporating risk factors of pharmacodynamics and pharmacokinetics in combination with RA can influence risks of hematoma development.

\section{Factor $\mathbf{X a}$ inhibitors Fondaparinux}

Investigators reported fondaparinux plasma $T_{1 / 2}$ of 17-21 hours with single-daily dosing and monitoring capability using anti-factor Xa activity; they performed only singleshot RA in conjunction with fondaparinux, and regional analagesic catheters were removed 2 hours before initial drug administration with first dose administered 6 hours postoperatively. ${ }^{28}$ Controlled conditions in this series with fondaparinux and neuraxial blockade revealed minimal evidence of hematoma formation. Fondaparinux can accumulate with renal dysfunction, and despite normal renal function, stable plateau requires 2-3 days to be achieved. Therefore, manufacturer recommends reducing dose with moderate renal insufficiency, and is contraindicated in those with severe renal insufficiency.

A study of 1,631 patients undergoing continuous neuraxial/ deep-PNB reported no serious hemorrhage, but catheters were removed 36 hours after last dose, and subsequent dosing delayed 12 hours following catheter removal. ${ }^{29}$ However, hematoma risk for patients receiving fondaparinux remains unknown, so management consensus statements are based on sustained and irreversible effect, dosing/timing, and report of one spinal hematoma during initial trials. Therefore, until further experience becomes available, performing deep-PNB/ neuraxial techniques should occur as single-needle passes, with atraumatic needle placement, avoidance of analgesic catheters, and avoiding RA with therapeutic dosing. Recent ASRA and ESRA consensus indicates a 3- to 4-day interval (ideally $5-T_{1 / 2}$ ) before performing RA procedures and then resuming medication 12-24 hours postprocedure. ${ }^{19}$

\section{Rivaroxaban}

An oral administered factor Xa inhibitor, with maximum effect in 1-4 hours, $T_{1 / 2}$ of 5-9 hours, administered once/day for thromboprophylaxis, first dose 6-8 hours postsurgery, but no antidote is available. Clinicians should adhere to regulatory recommendations and label inserts, particularly in clinical situations associated with increased risk of bleeding. Some evidence exists that patients may be monitored with antifactor Xa activity, prothrombin-time, and aPTT (activated partial thromboplastin time; shows linear dose effect). ${ }^{6,20}$ Investigations comparing rivaroxaban with LMWH demonstrated similar efficacy and rates of bleeding. ${ }^{30}$ Rivaroxaban is cleared by liver, gut, and kidney, but clearance time can be prolonged in the elderly (13 hours) secondary to decline of renal function (dose adjustment with renal insufficiency and contraindicated in severe liver disease). ${ }^{30}$

No information regarding needle placement/cathetermanagement was demonstrated during clinical trials despite lack of reported hematoma formation when RA was performed. ${ }^{31}$ As a new anticoagulant, along with lack of experience regarding RA performance and prolonged medication $T_{1 / 2}$, a cautious approach is warranted when planning neuraxial/deep-PNB. Therefore, as per ESRA guidelines, an interval of 22-26 hours between the last rivaroxaban dose and RA is recommended, and next dose administered 4-6 hours following catheter withdrawal. ${ }^{6,32}$ Recently published interim update to ASRA Anticoagulation (third edition) and recent ESRA/World Institute of Pain consensus recommend an interval of 3 days (roughly $5-T_{1 / 2}$ ) prior to RA and delaying drug administration 6 hours postprocedure. ${ }^{4,19}$

\section{Apixaban}

Orally administered and reversible direct factor Xa inhibitor, $T_{1 / 2} 10-15$ hours, elimination is $25 \%$ renal and $75 \%$ hepatic/ biliary with intestinal excretion. Lack of information and approved applications along with no consensus regarding risk assessment or patient management regarding $\mathrm{RA}$ is available. As with other untested thromboprophylactic medications in combination with RA, suggestions are to follow/delay a minimum $2-T_{1 / 2}$ before performing neuraxial/deep-PNB that 
would theoretically lessen medication efficacy and reduce risk of bleeding. This results in a time interval of 26-30 hours between last apixaban administration and catheter withdrawal, with next dose-delayed 6 hours.

An update to ASRA Anticoagulation (third edition) and recent consensus by ESRA, ASRA, and World Institute of Pain regarding apixaban and RA suggest a 3-5 day interval $\left(5-T_{1 / 2}\right)^{4,19}$ between last apixaban dose and deep-PNB/ neuraxial interventions. As experience with this agent is limited, along with wide-ranging pharmacokinetics of apixaban therapy, it is warranted to delay postprocedure administration by 6 hours. . $^{1,32}$

\section{Danaparoid}

Indirect factor $\mathrm{Xa}$ inhibitor with coagulation effects through antithrombin-mediated inhibition of factor Xa. It is a glycosaminoglycan mixture containing $84 \%$ heparin-sulfate resulting in $10 \%$ incidence/potential for HIT. ${ }^{33,34}$ It has a long $T_{1 / 2}$ (22 hours) that could be prolonged with renal insufficiency, so, dose adjustments are necessary and coagulation monitoring made possible by measuring anti-Xa activity. There are reports of severe bleeding, there is no antidote, and it cannot be hemofiltered, but can be removed using plasmapheresis. Thromboprophylaxis recommendations indicate that first dose be administered 2 hours preoperatively, then twice daily. Although neuraxial blockade was performed in a small number of patients during clinical trials, RA is not being recommended as significant plasma levels can be obtained with preoperative dosing. Instead, preoperative administration should be avoided if neuraxial/ deep-PNB anesthesia is planned along with avoiding use of catheters.

\section{Idrabiotaparinux}

Initial trials with idraparinux were abandoned due to major bleeding and were reformulated to idrabiotaparinux. ${ }^{35}$ It is a factor Xa inhibitor, has extremely long $T_{1 / 2}$ (once-weekly SQ injection), and clearance is mainly renal; therefore, accumulation warning(s) are to be given in elderly and those with renal insufficiency. Concern for potential rebound influence from tissue-site redistribution is warranted due to long drug $T_{1 / 2}$. Some trials have reported similar efficacy with less bleeding compared to warfarin. ${ }^{35}$ There are currently no data regarding performing perioperative RA, which is contraindicated, pending further investigation.

There are positive findings from clinical trials of an antidote which may reverse anti-factor Xa consequences of idrabiotaparinux. ${ }^{36}$ This antidote, avidin, could prove effective in reversing coagulation dysfunction caused by idrabiotaparinux, and despite its short $T_{1 / 2}$, rebound anticoagulation effect has not been observed in clinical trials. ${ }^{36}$

\section{Thrombin inhibitors}

These medications interrupt proteolysis properties of thrombin. Unlike heparin, thrombin inhibitors influence fibrin formation and inactivate fibrin already bound to thrombin (inhibiting further thrombus formation). These medications lack a specific antidote, but hirudins and argatroban can be removed with dialysis.

\section{Hirudins: desirudin, lepirudin, bivalirudin}

These recombinant hirudins are first generation direct thrombin inhibitors and are indicated for thromboprophylaxis (desirudin), prevention of DVT and pulmonary embolism (PE) after hip replacement, ${ }^{30}$ and DVT treatment (lepirudin) in patients with HIT. ${ }^{37}$ They are administered by the parenteral route, have an elimination $T_{1 / 2}$ of 30 minutes to 3 hours, can accumulate with renal insufficiency (ie, lepirudin dose reduction of $85 \%$ with severe renal impairment), and should be monitored using aPTT or ecarin clotting time (ECT; more specific). Prolonged aPTT is required for effective thromboprophylaxis, and following a single injection of desirudin, there is an increase in aPTT which is measurable within 30 minutes and reaches a maximum in 2 hours. There is still a prolonged aPTT 8 hours after SQ administration of low-dose hirudins. Lepirudin has been associated with antibody formation (incidence 40\%), delayed elimination, unpredictable and prolonged activity, as well as association with bleeding and anaphylaxis. ${ }^{38}$

In early clinical trials, desirudin was administered in a small number of patients undergoing neuraxial puncture without evidence of hematoma (single report of spontaneous epidural hematoma with lepirudin). Owing to lack of information and application(s) of these agents, no statement(s) regarding RA risk assessment and patient management can be made (HIT patients typically need therapeutic levels of anticoagulation making them poor candidates for RA). Therefore, no statement(s) regarding risk assessment and patient management can be made. Administration of thrombin inhibitors in combination with other antithrombotic agents should always be avoided. In those rare circumstances where RA would be planned, it is recommended to wait for a minimum of 8-10 hours following last dose (longer with renal insufficiency), along with evidence of aPTT or ECT within normal limits before proceeding with needle puncture, and then waiting/ observing/monitoring for at least 2-4 hours postprocedure 
before next dosing. However, secondary to potential bleeding issues and route of administration, the trend with these thrombin inhibitors has been to replace them with factor Xa inhibitors (ie, fondaparinux - DVT prophylaxis) or use of argatroban (factor IIa inhibitor) for acute HIT.

\section{Argatroban}

It is intravenously administered, reversible, and a direct thrombin inhibitor approved for management of acute HIT (type II). Advantages/uniqueness over other thrombin inhibitors include its elimination through the liver (indication in compromising renal dysfunction) and short $T_{1 / 2}$ (35-40 minutes) that reveals normalization of aPTT in 2-4 hours following discontinuation. However, dose reduction should be considered in critically ill and those with heart failure or impaired hepatic function.

\section{Dabigatran}

An oral thrombin inhibitor approved for thromboprophylaxis (similar efficacy to LMWH's and warfarin without increased risk of bleeding), prolongs aPTT (not linear), reversibly inhibits free- and clot-bound thrombin, with plasma levels peaking at $1-3$ hours, and has a $T_{1 / 2}$ of 8 hours with single-dose (12-15 hours with multiple doses). ${ }^{3}$ Initially, dosing was $220 \mathrm{mg} / \mathrm{d}$ with first dose of $110 \mathrm{mg} \mathrm{1-4} \mathrm{hours}$ postoperatively. ${ }^{6,20}$ In patients with renal compromise, dose suggested is $150 \mathrm{mg}$ for maintenance, with first dose of $75 \mathrm{mg}$ (contraindicated in renal failure). ${ }^{30}$ More recent dosing guidelines for atrial fibrillation indicated $150 \mathrm{mg}$ twice daily (normal renal function), and $110 \mathrm{mg}$ twice daily in patients with renal dysfunction (United States: $75 \mathrm{mg}$ twice daily with creatinine clearance of $15-30 \mathrm{~mL} / \mathrm{min}$ ), and $220 \mathrm{mg} / \mathrm{d}$ for thromboprophylaxis..$^{39}$ Published reports made no attempt to randomize patients with respect to RA or impose exclusion criteria if planning dabigatran administration. Single-shot RA has been performed with no reported hematoma events, but lack of information regarding specifics of RA performance along with prolonged $T_{1 / 2}$ warrants a cautious approach. The $T_{1 / 2}$ of dabigatran (12-15 hours in healthy patients) suggests an interval of 34 hours between last dose and catheter manipulation/withdrawal, but analgesic catheters are not recommended. ASRA Anticoagulation (third edition) interim update and the published consensus by ASRA, ESRA, and World Institute of Pain suggests waiting 4-5 days $\left(5-T_{1 / 2}\right)$ from last administration before performing RA, 6 hours to initiate medication post-RA and 6 hours between removal of neuraxial catheter and next dose. ${ }^{4,19,32}$

\section{Thrombolytics/fibrinolytics}

These agents dissolve clot(s) secondary to the action of plasmin. Plasminogen activators, streptokinase, and urokinase dissolve thrombus and influence plasminogen, leading to decreased levels of plasminogen and fibrin. Clot lysis elevates fibrin split/degradation products that also have an anticoagulant effect (inhibits platelet aggregation). Thrombolytic therapy will maximally depress fibrinogen and plasminogen for 5 hours following therapy and remain depressed for 27 hours. $^{40}$

Original recommendations to initiate thrombolytic therapy was contraindicated within 10 days following neuraxial/deep-PNB procedures and surgery, but in a recent consensus statement by ASRA and ESRA, it was reduced to 2 day minimum and performing assessments every 2 hours for neurological deficits. ${ }^{19}$ The 2-day minimum is based on prolonged plasminogen depression of 27 hours. ${ }^{19}$ Definitive data are not available on when to discontinue these agents and the safe time to neuraxial/deep-PNB placement, which ranges from 24 hours $^{41}$ to 10 days, ${ }^{40}$ but it should be noted that clots are not stable for 10 days postthrombolytic therapy. ${ }^{19}$ However, performing superficial PNB (compressible vessels or bleeding easily managed) earlier than 10 days can be evaluated with caution on an individual basis, weighing risk to benefit. There are no recommendations for removal of analgesic catheters for patients receiving fibrinolytic/ thrombolytic medications, but fibrinogen levels (later factor to recover) can provide relative guidance on thrombolytic effect and timing of catheter removal.

\section{Discussion}

Perioperative thromboembolism is a health care issue, source of morbidity, and nearly all hospitalized patients have risk factor(s) for such an event (at least one), with approximately $40 \%$ of patients having three or more risk factors (Table 2). ${ }^{42,43}$ Improvement in perioperative outcomes have been demonstrated with $\mathrm{RA}^{44-47}$ by decreasing morbidity and mortality due to attenuation of hypercoagulable response from surgery and reduction in thromboembolism frequency. Despite such beneficial effects, regional techniques alone prove insufficient as the sole method of thromboprophylaxis. As a result, hospitalized patients become candidates for thromboprophylaxis, and perioperative anticoagulant, antiplatelet, and thrombolytic medications are increasingly used for prevention and treatment (Table 3).

Thromboembolism remains a source of perioperative compromise, yet its prevention and treatment are also associated with risk. Bleeding can occur with prophylactic 
Table 2 Risk factors for perioperative thromboembolism in hospitalized patients

\begin{tabular}{ll}
\hline Patient-specific risks & Perioperative-related risks \\
\hline Cancer & Trauma \\
Venous compression (tumor, & Surgery (especially major \\
hematoma) & surgery) \\
Increasing age & Lower extremity injuries \\
History of previous VTE & Immobility \\
Acute medical illness & Cancer therapy (hormonal, \\
& chemo) \\
Pregnancy & Radiotherapy \\
Nephrotic syndrome & Lower extremity paresis \\
Inflammatory bowel disease & Estrogen-containing oral \\
& contraceptives \\
Obesity & Selective estrogen receptor \\
& modulators \\
Myeloproliferative disorders & Indwelling neuraxial catheter \\
Inherited/acquired thrombophilia & Erythropoiesis-stimulating \\
& agents \\
Abnormal spinal cord/vertebral column & Central venous catheter \\
& $\begin{array}{l}\text { placement } \\
\text { Sustained anticoagulation }\end{array}$ \\
Organ dysfunction (especially renal & \\
disease) & Errors in VTE prophylaxis \\
Sex (higher risks in females) & Hip or knee arthroplasty \\
Spinal cord injury & High- versus low-risk surgery \\
Sick medical patients requiring bed rest & Traumatic needle/catheter \\
Hormone replacement therapy & placement \\
\hline
\end{tabular}

Abbreviation: VTE, venous thromboembolism.

and therapeutic anticoagulation as well as thrombolytic therapy. Intracranial, intraspinal, intraocular, mediastinal, or retroperitoneal bleeding are classified as major; bleeding that leads to morbidity, results in hospitalization, or requires transfusion is also considered major. Risk factors for bleeding during anticoagulation include intensity of anticoagulant effect, increased age, female sex, history of gastrointestinal bleeding, concomitant anticoagulant use, and duration of therapy. ${ }^{48}$ Fluctuation(s) in anticoagulant effect increases the likelihood of a serious bleed. In a case-control study, risk of intracranial hemorrhage doubled for each increase of approximately 1 in the INR. ${ }^{48}$ Incidence of hemorrhagic complications is greatest with thrombolytic therapy, with major hemorrhage occurring in 6\%-30\% of patients treated (ie, thrombolytic therapy for DVT, ischemic stroke, or ST elevation myocardial infarction) ${ }^{48}$

Frequently encountered perioperative events are in patients on long-term anticoagulation (history of DVT/PE, mechanical heart valves, atrial fibrillation, etc) who present for elective or urgent surgery. This is a situation where riskto-benefit analyses must be performed when considering RA, as minor procedures do not require interruption of therapy, whereas continuation of coagulation-altering medications in setting of major surgery increases bleeding risks. Thus, perioperative management involves balancing risks of surgical bleeding versus thromboembolism, whether interruption of antithrombotic/antiplatelet therapy is necessary and/or any requirements for "bridging" therapy. Perioperative management guidelines of antithrombotic therapy in such situations have been addressed by the $\mathrm{ACCP}^{49}$ and summarized in Table 4 , but complexity arises during perioperative planning in determining who is at risk and determining whether or not to perform $\mathrm{RA}^{50}$ as well as types of surgeries considered low-to-high risk.

Neurologic dysfunction from hemorrhagic complications of RA is unknown, but is suggested to be higher than previously reported and increasing in frequency. ${ }^{50}$ As a result, risks and safety issues for patient's receiving antithrombotic therapy has increased, especially in certain patient populations. ${ }^{51}$ Publications on large series of uneventful peripheral blockade in combination with antithrombotic therapy and case reports of hemorrhagic complications following peripheral techniques have influenced evidence-based recommendations. Such results revealed that risks of clinically significant bleeding increases with age, abnormalities of the spinal cord or vertebral column (during neuraxial RA), presence of an underlying coagulopathy, difficulty during RA needle placement, from an indwelling catheter during sustained anticoagulation and a host of surgery-specific circumstances (immobility, cancer therapy, etc). Therefore, vigilance, prompt diagnosis, and intervention are required to eliminate, reduce, and optimize neurologic outcome should clinically significant bleeding occur.

Anticoagulant and thromboprophylactic medications and duration of administration should be based on identification of individual- and group-specific risk factors (Tables 2 and 4). Individualized approach(s) alone to thromboprophylaxis proves to be complex and not routinely applied, so recommendations are by default group specific. However, as newer thromboprophylactic agents are introduced, additional complexity into the guidelines (duration of therapy, degree of anticoagulation) and consensus management must also evolve. ${ }^{42,52}$ Yet, recommendations from the Eighth ACCP Guidelines revealed that reductions of clinically relevant events have been difficult to identify. ${ }^{53}$ Some reasons may be lack of adherence to balance between thromboembolic complications and bleeding, differences in populations (control groups versus actual patients), use of surrogate end points, and early studies that did not appreciate patients at increased risk for surgical bleeding. Therefore, establishment of overall risk-to-benefit ratio of antithrombotic therapy during surgery 


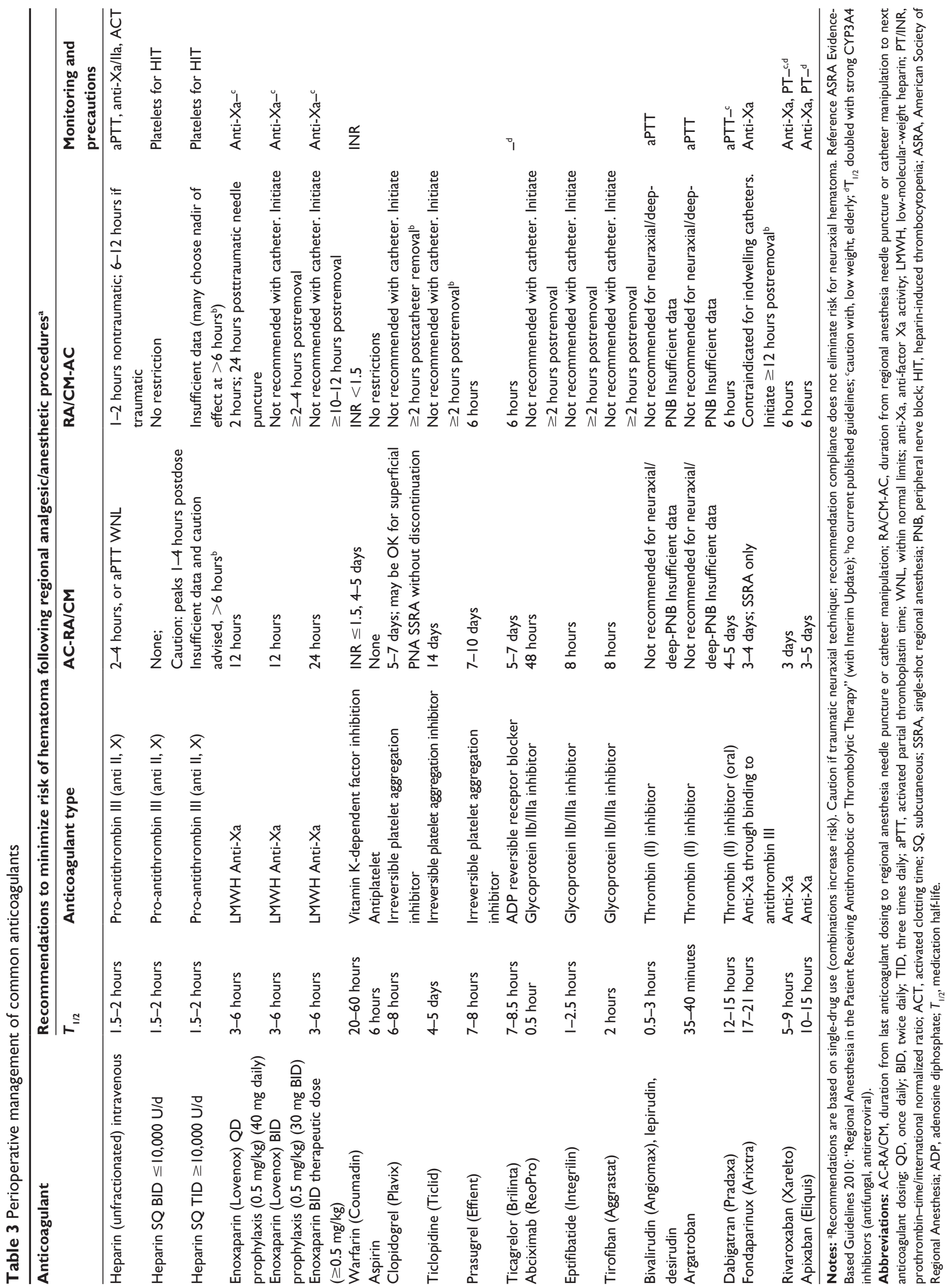


Table 4 Risks stratification, perioperative management, and chemoprophylaxis

Standard risk of both DVT/PE and major bleeding

- Aspirin: 325 mg, two times per day, reduce with Gl dysfunction, start day of surgery

- LMWH: dose-per packing insert, 12-24 hours postop or following N cath removal

- Warfarin: goal INR $\leq 2.0$, start night before/after surgery

- Pentasaccharides: dose-per packing insert, 12-24 hours postop or after $\mathrm{N}$ cath removal

Standard risk of DVT/PE and increased risk of major bleeding

- Aspirin: 325 mg, two times per day, reduce with Gl dysfunction, start day of surgery

- Warfarin: goal INR $\leq 2.0$, start night before/after surgery

- No chemoprophylaxis

Elevated risk of DVT/PE and standard risk of major bleeding

- LMWH: dose-per packing insert, 12-24 hours postop or following $N$ cath removal

- Warfarin: goal INR $\leq 2.0$, start night before/after surgery

- Pentasaccharides: dose-per packing insert, 12-24 hours postop or after $\mathrm{N}$ cath removal

Elevated risk of both DVT/PE and major bleeding

- Aspirin: $325 \mathrm{mg}, \mathrm{BID}$, reduce with GI dysfunction, start day of surgery

- Warfarin: goal INR $\leq 2.0$, start night before/after surgery

- No chemoprophylaxis

Patients on antiplatelet therapy

Patients at low risk of cardiac events

- D/C antiplatelet Tx 7-10 days prior to surgery

- Resume Tx 24 hours postop

Patients at high risk of cardiac events (not considering cardiac/coronary stents)

- Aspirin continued throughout periop period

- $\mathrm{D} / \mathrm{C}$ thienopyridines before surgery (clopidogrel 10 day/at least 5 days prior)

- Restart clopidogrel 24 hours postoperatively

Patients with coronary stents along with aspirin + thienopyridine $T x$

I. Postpone elective surgery for following duration(s):

- Bare-metal stent(s) - 4-6 weeks

- Drug-eluting stent(s) - 12 months

2. Surgery cannot be postponed. Emergency/urgent surgery

- Continue aspirin during perioperative period

Abbreviations: BID, twice daily; Cath, catheter; D/C, discontinue; DVT/PE, deep vein thrombosis/pulmonary embolism; LMWH, low-molecular-weight heparin; $\mathrm{GI}$, gastrointestinal; INR, international normalized ratio; $\mathrm{N}$, neuraxial; postop, postoperatively; Tx, treatment/therapy.

and performing deep-PNB/neuraxial blockade must be made on an individual basis, observing published guidelines and consensus statements to mitigate confusion, especially in conjunction with newer NOACs.

\section{Conclusion}

Several NOACs offer oral routes of administration, simple dosing regimen, efficacy with less bleeding risks, reduced requirement for clinical monitoring, and alternative elimination mechanisms other than renal. Anticoagulant and thrombolytic combination therapy has additive or synergistic effect requiring dose adjustment(s) based on patient-specific (renal, hepatic, cardiac condition) and surgery-related (trauma, cancer, etc) issues to safely administer RA. Anticoagulant/thrombolytic antidotes are rare or show slow clinical effects (eg, vitamin K slowly reverses function of anti-vitamin $\mathrm{K}$ anticoagulants), and therapies including dialysis (remove thrombin inhibitor hirudins and argatroban), plasmapheresis (removes indirect factor Xa inhibitor danaparoid), and fresh-frozen plasma (to reduce fluid overload, supplement coagulation factors with specific factor therapy) can prove useful in certain situations.

Due to confusion while mixing RA with prophylactic and therapeutic coagulation-altering medications, understanding of complexity is essential and a "cookbook" approach is not appropriate intervention. Instead, decisions about RA in patients receiving antithrombotic/prophylactic therapy must be made on an individual basis depending on 1) risk of neuraxial or deep peripheral hematoma development with benefits of RA; 2) following manufacturer-suggested dosing guidelines; ${ }^{48}$ 3) alternative anesthetic/analgesic techniques for patients with unacceptable risks; 4) coagulation status optimization at time of neuraxial/deep peripheral needle/catheter placement and anticoagulation level being monitored during analgesic catheterization; 5) indwelling catheters never being removed under therapeutic anticoagulation; and 6) understanding that merely identifying risk factors and following consensus-guidelines will not completely eliminate hematoma formation or complications from nerve tissue compression. Therefore, maximizing patient-specific thromboprophylaxis along with recognition of group-specific and surgery-related risks remain important.

\section{Disclosure}

The authors report no conflicts of interest in this work.

\section{References}

1. Gomez-Outes A, Avendano-Sola C, Terleira-Fernandez AI, VargasCastrillon E. Pharmacoeconomic evaluation of dabigatran, rivaroxaban and apixaban versus enoxaparin for the prevention of venous thromboembolism after total hip or knee replacement in Spain. Pharmacoeconomics. 2014;32(9):919-936.

2. Vílchez JA, Gallego P, Lip GY. Safety of new oral anticoagulant drugs: a perspective. Ther Adv Drug Saf. 2014;5(1):8-20.

3. Baglin T. Clinical use of new oral anticoagulant drugs: dabigatran and rivaroxaban. Br J Haematol. 2013;163(2):160-167.

4. Horlocker TT, Wedel DJ, Rowlingson JC, Enneking FK, Physicians ACoC. Executive summary: regional anesthesia in the patient receiving antithrombotic or thrombolytic therapy: American Society of Regional Anesthesia and Pain Medicine Evidence-Based Guidelines (Third Edition). Reg Anesth Pain Med. 2010;35(1):102-105.

5. Tryba M. European practice guidelines: thromboembolism prophylaxis and regional anesthesia. Reg Anesth Pain Med. 1998;23(6): $178-182$.

6. Gogarten W, Vandermeulen E, Van Aken H, et al. Regional anaesthesia and antithrombotic agents: recommendations of the European Society of Anaesthesiology. Eur J Anaesthesiol. 2010;27(12):999-1015. 
7. Rosencher N, Bonnet MP, Sessler DI. Selected new antithrombotic agents and neuraxial anaesthesia for major orthopaedic surgery: management strategies. Anaesthesia. 2007;62(11):1154-1160.

8. Shireman TI, Howard PA, Kresowik TF, Ellerbeck EF. Combined anticoagulant-antiplatelet use and major bleeding events in elderly atrial fibrillation patients. Stroke. 2004;35(10):2362-2367.

9. Verheugt FW. Combined antiplatelet and novel oral anticoagulant therapy after acute coronary syndrome: is three a crowd? Eur Heart J. 2013;34(22):1618-1620.

10. Benzon HT, Wong HY, Siddiqui T, Ondra S. Caution in performing epidural injections in patients on several antiplatelet drugs. Anesthesiology. 1999;91(5):1558-1559.

11. Horlocker TT, Wedel DJ, Benzon H, et al. Regional anesthesia in the anticoagulated patient: defining the risks (the second ASRA Consensus Conference on Neuraxial Anesthesia and Anticoagulation). Reg Anesth Pain Med. 2003;28(3):172-197.

12. Buvanendran A, Young AC. Spinal epidural hematoma after spinal cord stimulator trial lead placement in a patient taking aspirin. Reg Anesth Pain Med. 2014;39(1):70-72.

13. Sarubbo S, Garofano F, Maida G, Fainardi E, Granieri E, Cavallo MA. Spontaneous and idiopathic chronic spinal epidural hematoma: two case reports and review of the literature. Eur Spine J. 2009;18(11): 1055-1061.

14. Dimou J, Jithoo R, Morokoff A. Spontaneous spinal epidural haematoma in a geriatric patient on aspirin. J Clin Neurosci. 2010;17(1): $142-144$.

15. Cappelleri JC, Fiore LD, Brophy MT, Deykin D, Lau J. Efficacy and safety of combined anticoagulant and antiplatelet therapy versus anticoagulant monotherapy after mechanical heart-valve replacement: a metaanalysis. Am Heart J. 1995;130(3 Pt 1):547-552.

16. Johnson SG, Rogers K, Delate T, Witt DM. Outcomes associated with combined antiplatelet and anticoagulant therapy. Chest. 2008;133(4): 948-954.

17. Moon HJ, Kim JH, Kwon TH, Chung HS, Park YK. Spontaneous spinal epidural hematoma: an urgent complication of adding clopidogrel to aspirin therapy. J Neurol Sci. 2009;285(1-2):254-256.

18. Leese PT, Hubbard RC, Karim A, Isakson PC, Yu SS, Geis GS. Effects of celecoxib, a novel cyclooxygenase-2 inhibitor, on platelet function in healthy adults: a randomized, controlled trial. J Clin Pharmacol. 2000;40(2):124-132.

19. Narouze S, Benzon HT, Provenzano DA, et al. Interventional spine and pain procedures in patients on antiplatelet and anticoagulant medications: guidelines from the american society of regional anesthesia and pain medicine, the European society of regional anaesthesia and pain therapy, the american academy of pain medicine, the international neuromodulation society, the north american neuromodulation society, and the world institute of pain. Reg Anesth Pain Med. 2015;40(3): $182-212$.

20. Horlocker TT. Regional anaesthesia in the patient receiving antithrombotic and antiplatelet therapy. Br J Anaesth. 2011;107(Suppl 1):i96-i106.

21. Davis JJ, Bankhead BR, Eckman EJ, Wallace A, Strunk J. Three-timesdaily subcutaneous unfractionated heparin and neuraxial anesthesia: a retrospective review of 928 cases. Reg Anesth Pain Med. 2012;37(6): 623-626.

22. Warkentin TE, Levine MN, Hirsh J, et al. Heparin-induced thrombocytopenia in patients treated with low-molecular-weight heparin or unfractionated heparin. N Engl J Med. 1995;332(20):1330-1335.

23. Junqueira DR, Perini E, Penholati RR, Carvalho MG. Unfractionated heparin versus low molecular weight heparin for avoiding heparininduced thrombocytopenia in postoperative patients. Cochrane Database Syst Rev. 2012;9:CD007557.

24. Ang E. Unfractionated heparin versus low-molecular-weight heparin for avoiding heparin-induced thrombocytopenia in postoperative patients. Clin Nurse Spec. 2013;27(5):229-230.

25. Hirsh J, Bauer KA, Donati MB, et al. Parenteral anticoagulants: American College of Chest Physicians Evidence-Based Clinical Practice Guidelines (8th Edition). Chest. 2008;133(6 Suppl):141S-159S.
26. van Veen JJ, Maclean RM, Hampton KK, et al. Protamine reversal of low molecular weight heparin: clinically effective? Blood Coagul Fibrinolysis. 2011;22(7):565-570.

27. Ansell J, Hirsh J, Hylek E, et al. Pharmacology and management of the vitamin K antagonists: American College of Chest Physicians Evidence-Based Clinical Practice Guidelines (8th Edition). Chest. 2008; 133(6 Suppl):160S-198S.

28. Turpie AG, Gallus AS, Hoek JA, Pentasaccharide I. A synthetic pentasaccharide for the prevention of deep-vein thrombosis after total hip replacement. N Engl J Med. 2001;344(9):619-625.

29. Singelyn FJ, Verheyen CC, Piovella F, Van Aken HK, Rosencher N; EXPERT Study Investigators. The safety and efficacy of extended thromboprophylaxis with fondaparinux after major orthopedic surgery of the lower limb with or without a neuraxial or deep peripheral nerve catheter: the EXPERT Study. Anesth Analg. 2007;105(6):1540-1547, table of contents.

30. Eriksson BI, Quinlan DJ, Weitz JI. Comparative pharmacodynamics and pharmacokinetics of oral direct thrombin and factor xa inhibitors in development. Clin Pharmacokinet. 2009;48(1):1-22.

31. Lassen MR, Ageno W, Borris LC, et al. Rivaroxaban versus enoxaparin for thromboprophylaxis after total knee arthroplasty. $N$ Engl J Med. 2008;358(26):2776-2786.

32. Horlocker TT, Wedel DJ, Rowlingson JC, et al. Regional anesthesia in the patient receiving antithrombotic or thrombolytic therapy: American Society of Regional Anesthesia and Pain Medicine Evidence-Based Guidelines (Third Edition). Reg Anesth Pain Med. 2010;35(1):64-101.

33. Naeshiro N, Aikata H, Hyogo H, et al. Efficacy and safety of the anticoagulant drug, danaparoid sodium, in the treatment of portal vein thrombosis in patients with liver cirrhosis. Hepatol Res. 2015;45(6):656-662.

34. Tardy-Poncet B, Combe M, Piot M, Chapelle C, Akrour M, Tardy B. Effects of argatroban, danaparoid, and fondaparinux on trombin generation in heparin-induced thrombocytopenia. Thromb Haemost. 2013;109(3):504-509.

35. Harenberg J, Vukojevic Y, Mikus G, Joerg I, Weiss C. Long elimination half-life of idraparinux may explain major bleeding and recurrent events of patients from the van Gogh trials. J Thromb Haemost. 2008;6(5): 890-892.

36. Paty I, Trellu M, Destors JM, Cortez P, Boelle E, Sanderink G. Reversibility of the anti-FXa activity of idrabiotaparinux (biotinylated idraparinux) by intravenous avidin infusion. J Thromb Haemost. 2010;8(4):722-729.

37. Greinacher A, Lubenow N. Recombinant hirudin in clinical practice: focus on lepirudin. Circulation. 2001;103(10):1479-1484.

38. Linnemann B, Greinacher A, Lindhoff-Last E. Alteration of pharmacokinetics of lepirudin caused by anti-lepirudin antibodies occurring after long-term subcutaneous treatment in a patient with recurrent VTE due to Behcets disease. Vasa. 2010;39(1):103-107.

39. Levy JH, Faraoni D, Spring JL, Douketis JD, Samama CM. Managing new oral anticoagulants in the perioperative and intensive care unit setting. Anesthesiology. 2013;118(6):1466-1474.

40. Rosenquist RW, Brown DL. Neuraxial bleeding: fibrinolytics/thrombolytics. Reg Anesth Pain Med. 1998;23(6 Suppl 2):152-156.

41. Breivik H, Bang U, Jalonen J, Vigfusson G, Alahuhta S, Lagerkranser M. Nordic guidelines for neuraxial blocks in disturbed haemostasis from the Scandinavian Society of Anaesthesiology and Intensive Care Medicine. Acta Anaesthesiol Scand. 2010;54(1):16-41.

42. Geerts WH, Bergqvist D, Pineo GF, et al. Prevention of venous thromboembolism: American College of Chest Physicians EvidenceBased Clinical Practice Guidelines (8th Edition). Chest. 2008; 133(6 Suppl):381S-453S.

43. Huo MH, Spyropoulos AC. The eighth American college of chest physicians guidelines on venous thromboembolism prevention: implications for hospital prophylaxis strategies. J Thromb Thrombolysis. 2011;31(2): 196-208.

44. Capdevila X, Barthelet Y, Biboulet P, Ryckwaert Y, Rubenovitch J, d'Athis F. Effects of perioperative analgesic technique on the surgical outcome and duration of rehabilitation after major knee surgery. Anesthesiology. 1999;91(1):8-15. 
45. Liu S, Carpenter RL, Neal JM. Epidural anesthesia and analgesia. Their role in postoperative outcome. Anesthesiology. 1995;82(6): 1474-1506.

46. Rodgers A, Walker N, Schug S, et al. Reduction of postoperative mortality and morbidity with epidural or spinal anaesthesia: results from overview of randomised trials. BMJ. 2000;321(7275):1493.

47. Tuman KJ, McCarthy RJ, March RJ, DeLaria GA, Patel RV, Ivankovich AD. Effects of epidural anesthesia and analgesia on coagulation and outcome after major vascular surgery. Anesth Analg. 1991; 73(6):696-704.

48. Schulman S, Beyth RJ, Kearon C, Levine MN, American College of Chest P. Hemorrhagic complications of anticoagulant and thrombolytic treatment: American College of Chest Physicians EvidenceBased Clinical Practice Guidelines (8th Edition). Chest. 2008; 133(6 Suppl):257S-298S.

49. Douketis JD, Berger PB, Dunn AS, et al. The perioperative management of antithrombotic therapy: American College of Chest Physicians Evidence-Based Clinical Practice Guidelines (8th Edition). Chest. 2008;133(6 Suppl):299S-339S.
50. Dowling NF, Beckman MG, Manco-Johnson M, et al. The US Thrombosis and Hemostasis Centers pilot sites program. JThromb Thrombolysis. 2007;23(1):1-7.

51. Bates SM, Greer IA, Hirsh J, Ginsberg JS. Use of antithrombotic agents during pregnancy: the Seventh ACCP Conference on Antithrombotic and Thrombolytic Therapy. Chest. 2004;126(3 Suppl):627S-644S.

52. ACCP-NHLBI National Conference on Antithrombotic Therapy. American College of Chest Physicians and the National Heart, Lung and Blood Institute. Chest. 1986;89(2 Suppl):1S-106S.

53. Mantilla CB, Horlocker TT, Schroeder DR, Berry DJ, Brown DL. Frequency of myocardial infarction, pulmonary embolism, deep venous thrombosis, and death following primary hip or knee arthroplasty. Anesthesiology. 2002;96(5):1140-1146.

\section{Publish your work in this journal}

Local and Regional Anesthesia is an international, peer-reviewed, open access journal publishing on the development, pharmacology, delivery and targeting and clinical use of local and regional anesthetics and analgesics. The journal welcomes submitted papers covering original research, basic science, clinical studies, reviews \& evaluations,

\section{Dovepress}

guidelines, expert opinion and commentary, case reports and extended reports. The manuscript management system is completely online and includes a very quick and fair peer-review system, which is all easy to use. Visit http://www.dovepress.com/testimonials.php to read real quotes from published authors. 